\title{
Avaliação do estilo de vida de hipertensos no Município de Malta-PB
}

\section{Lifestyle assessment in hypertensive Municipality of Malta-PB}

\author{
Juliana de Almeida Leandro Bezerra \\ Enfermeira formada pelas Faculdades Integradas de Patos \\ Gisele Santana Pereira Carreiro \\ Docente das Faculdades Integradas de Patos \\ Anne Milane Formiga Bezerra \\ Enfermeira docente das Faculdades Integradas de Patos. E-mail: annemilane_pb@hotmail.com \\ Kaaio Rodrigo Santos Bezerra \\ Bacharelado em Educação Física pelas Faculdades Integradas de Patos \\ Kévia Katiúcia Santos Bezerra \\ Médica Ginecologista e Obstetra Docente da UFCG campus Cajazeiras-PB
}

\begin{abstract}
Resumo: A Hipertensão Arterial Sistêmica representa um grave problema de saúde pública no país, sendo um dos fatores de risco para doenças cardiovasculares. É caracterizado pela elevação da pressão arterial a nível igual ou superior a $140 \mathrm{mmHg}$ da pressão sistólica $90 \mathrm{mmHg}$ da diastólica. Este estudo tem por objetivo verificar o estilo de vida dos portadores de Hipertensão Arterial atendidos em uma unidade de saúde da família de um município paraibano. Caracteriza-se por um estudo do tipo exploratório, descritivo, com abordagem quantitativa e qualitativa. A amostra do estudo foi constituída por 30 hipertensos. Os dados apresentados em tabelas e gráficos revelaram que a maioria dos hipertensos está em idade superior a 60 anos, cumpre algumas das orientações transmitidas pelo profissional de saúde, como a não ingestão de sal, não ingestão de gordura, prática de atividade física e não uso do álcool e do tabaco. Verificou-se também que todos os entrevistados fazem uso de medicamentos no tratamento da Hipertensão Arterial. Foi observado um estilo de vida um pouco acomodado por parte dos entrevistados quanto ao uso de sal, de gordura e da prática de atividade física onde relataram não essas orientações. Isso nos leva a entender que estilo de vida da população está contribuindo para a elevação dos níveis tensionais e não haja um controle da hipertensão arterial.
\end{abstract}

Palavras-chave: Cuidado. Hipertensão. Tratamento

\begin{abstract}
The Hypertension is a major public health problem in the country, one of the risk factors for cardiovascular disease. It is characterized by elevated blood pressure level equal to or greater than $140 \mathrm{mmHg}$ systolic blood pressure $90 \mathrm{~mm} \mathrm{Hg}$ diastolic. This study aims to determine the lifestyle of patients with arterial hypertension treated at a health facility of the family of Paraiba municipality. It is characterized by a study of exploratory, descriptive, with quantitative and qualitative approach. The study sample consisted of 30 hypertensive. The data presented in tables and graphs showed that most hypertensive patients is older than 60, meets some of the guidelines passed by the health professional, such as no salt intake, not fat intake, physical activity and not using alcohol and tobacco. It was also found that all respondents make use of medications in the treatment of Hypertension. a lifestyle somewhat accommodated by the respondents regarding the use of salt, fat and physical activity which reported no such guidelines were observed. This leads us to understand that the lifestyle of the population is contributing to the elevation of blood pressure and there is no control of hypertension.
\end{abstract}

Keyword: Caution. Hypertension. Treatment 


\section{INTRODUÇÃO}

A hipertensão arterial sistêmica (HAS) representa grave problema de saúde pública no país, não só pela elevada prevalência, como também pelos casos não diagnosticados, não tratados de forma adequada ou ainda pelo abandono do tratamento. No Brasil, as doenças cardiovasculares são responsáveis por $33 \%$ dos óbitos com causas conhecidas. Além disso, essas doenças foram a primeira causa de hospitalização do setor público, entre 1996 e 1999, e responderam por $17 \%$ das internações de pessoas de 40 a 59 anos e $29 \%$ daquelas com 60 anos ou mais (PASSOS et al., 2006).

Segundo Smeltzer e Bare (2006) a hipertensão arterial (HA) é uma síndrome clínica, que é caracterizada pela elevação da pressão arterial a nível igual ou superior à $140 \mathrm{mmHg}$ da pressão sistólica e $90 \mathrm{mmHg}$ da diastólica, em pelo menos duas aferições subsequentes e em condições de repouso. A hipertensão arterial é uma condição clínica multifatorial caracterizada por níveis elevados e sustentados de pressão arterial (VI DIRETRIZES DE HIPERTENSÃO ARTERIAL, 2010). Na maioria dos casos, é uma doença silenciosa, entretanto, pode ser fatal. Quando ocorrem sintomas, já são provenientes de complicações.

Vários estudos mostram que existem alguns fatores considerados como de risco, que associados entre si a outras condições, favorecem ao aparecimento da hipertensão arterial, sendo eles: idade, sexo, antecedentes familiares, raça, obesidade, estresse, sedentarismo, álcool, tabaco, anticoncepcionais e alimentos com sódio e gordura (PESSUTO; CARVALHO, 1998). Diante da diversidade de fatores que contribuem para o favorecimento da doença, o tratamento deve consistir em uma associação entre a mudança do estilo de vida e tratamento medicamentoso apropriado (VICTOR, 2005).

Para Woods, Froelicher e Motzer (2005) é papel dos profissionais de saúde, diagnosticar a situação do paciente e informar sobre a HAS como patologia e um dos fatores de risco para doenças cardiovasculares, prescrever um tratamento, conforme a situação de cada paciente e avaliar seus efeitos.

Este estudo tem como objetivo verificar o estilo de vida dos portadores de hipertensão arterial de uma unidade de Saúde da Família no município na cidade de Malta-PB.

\section{METODOLOGIA}

Trata-se de uma pesquisa do tipo exploratória e descritiva, com abordagem quantiqualitativa. Para realização da pesquisa, utilizou-se como campo de estudo uma Unidade de Saúde da Família da cidade de Malta-PB, localizada no sertão da Paraíba, a 335 km da capital João Pessoa. A unidade atende a famílias da zona urbana e rural totalizando 652 famílias, no qual 240 pessoas são cadastradas como hipertensas. A população foi composta por 240 hipertensos cadastrados na unidade. A amostra foi constituída por 30 hipertensos, que após esclarecimento dos objetivos da pesquisa se dispuseram a participar, mediante assinatura do Termo de Consentimento Livre e Esclarecido, obedecendo aos seguintes critérios de inclusão: ser cadastrado na referida unidade, possuir condições cognitivas de responder ao questionário, e como critérios de exclusão: ser cadastrado fora da área de abrangência de unidade e não ser hipertenso.

O instrumento utilizado foi um questionário, composto por 17 questões divididas em duas partes: a primeira referindo-se aos dados socioeconômicos dos entrevistados e, a segunda relacionada aos dados específicos da Hipertensão Arterial. Os dados foram coletados no mês de novembro de 2010. Cada entrevista teve duração em média de 15 minutos e foi realizada individualmente em um ambiente tranquilo para que os entrevistados se sentissem à vontade em responder as perguntas.

Durante todo o curso da pesquisa foram considerados os aspectos éticos da Resolução nº 466/2012 do Conselho Nacional de Saúde, que trata de pesquisas envolvendo seres humanos no Brasil, e também assegura a garantia de que a privacidade do sujeito será preservada. Todos os participantes tomaram conhecimento dos objetivos da referida pesquisa, e também foi explicado que teriam a liberdade de escolha quanto a sua participação, garantia do anonimato e sair da pesquisa segundo sua vontade. A coleta de dados ocorreu após aprovação deste estudo pelo Comitê de Ética em Pesquisa das Faculdades Integradas de Patos - CEP/FIP.

\section{RESULTADOS E DISCUSSÃO}

Observa-se na tabela 1 que de um total de 30 entrevistados, 25 (83\%) são do sexo feminino e 5 (17\%) são do sexo masculino. A faixa etária desses entrevistados ficou compreendida entre 20 a 92 anos, tendo maior prevalência em indivíduos acima de 60 anos. Esses resultados apontam um alto índice de hipertensão no sexo feminino. Essa característica é confirmada por Woods, Froelicher, Motzer (2005), no momento que revelam que os homens tendem a ser mais hipertensos que as mulheres até a sétima década, onde a partir daí há uma maior prevalência entre as mulheres. Isso também pode ser explicado pelo fato de que as mulheres procuram o serviço de saúde com mais frequência do que os homens. Além disso, as mulheres também admitem mais facilmente que estão doentes. Por isso, há uma preocupação crescente com a saúde do homem.

Conforme as V Diretrizes Brasileiras de Hipertensão Arterial (2006), a prevalência global de hipertensão entre homens (26,6\%; IC 95\% 26,0-27,2\%) e mulheres (26,1\%; IC $95 \% 25,5-26,6 \%)$ insinua que o sexo não é um fator de risco para hipertensão. Só que, segundo a mesma diretriz, estimativas globais sugerem taxas de hipertensão mais elevadas para homens até 50 anos e para mulheres a partir da sexta década.

Segundo Marques (2007) atualmente o país concentra 15 milhões de pessoas com mais de 60 anos, o que demonstra grande preocupação pois, sabe-se que a incidência de hipertensão arterial é bem maior nessa faixa etária, chegando a atingir cerca 30 milhões de pessoas idosas.

Quanto a escolaridade, observa-se que $7(23 \%)$ são analfabetos, $2(7 \%)$ tem ensino fundamental completo, $13(43 \%)$ tem o ensino fundamental incompleto, $5(17 \%)$ 
concluíram o ensino médio, 1 (3\%) tem o ensino médio incompleto e 2 (7\%) concluiu o ensino superior. Notamos que o nível escolar da maioria dos entrevistados é relativamente baixo, o que pode interferir, diretamente, no conhecimento sobre a doença e na compreensão das orientações. Para Castro et al (2003) , indivíduos que completam o $3^{\circ}$ grau tem prevalência de $40 \%$ menor de hipertensão do que os que possuem menos escolaridade.

Quanto à renda familiar, observa-se que: $3(10 \%)$ possuem renda menor que um salário mínimo; 17 (57\%) possuem recebem até um salário mínimo e 10 (33\%) sobrevivem com mais de um salário mínimo. Em relação à ocupação, 19 dos entrevistados (63\%) são aposentados, 4 (13\%) são comerciantes, 2 (7\%) são autônomos, 3 (10\%) são do lar, $1(3,5 \%)$ é professora e $1(3,5 \%)$ é agente comunitário de saúde.
Os dados obtidos confirmam que a maioria dos entrevistados são aposentados e que sobrevivem com uma renda baixa, o que pode influenciar no tratamento, pois muitas vezes, os medicamentos não são fornecidos pela rede pública, tendo o usuário que arcar com as despesas. Além disso, esse fato pode dificultar a mudança do estilo de vida, principalmente no que se refere à alimentação.

Woods, Froelicher, Motzer (2005), correlacionam as barreiras sociais e financeiras com a PA, considerando que aqueles que possuem uma alto estilo de vida, apresentam baixo risco para HAS.

Segundo Lima MT, et al (2004), o fator sócio econômico impede a liberdade do paciente escolher o alimento adequado à sua dieta, obrigando-o a se adequar à hábitos alimentares comum na vida desses sujeitos.

Tabela 1: Distribuição da amostra segundo as variáveis: sexo, idade, escolaridade, renda familiar e ocupação MALTA - PB.

\begin{tabular}{|c|c|c|c|}
\hline \multicolumn{2}{|c|}{ Variáveis } & $\mathbf{N}^{\mathbf{o}}$ & $\%$ \\
\hline \multirow{3}{*}{ Sexo } & Feminino & 25 & 83 \\
\hline & Masculino & 05 & 17 \\
\hline & Total & 30 & 100 \\
\hline \multirow{4}{*}{ Idade } & 20 a 40 anos & 04 & 13 \\
\hline & 41 a 60 anos & 08 & 27 \\
\hline & Acima de 60 anos & 18 & 60 \\
\hline & Total & 30 & 100 \\
\hline \multirow{8}{*}{ Escolaridade } & Analfabeto & 07 & 23 \\
\hline & Fund. Completo & 02 & 07 \\
\hline & Fund. Incompleto & 13 & 43 \\
\hline & Méd. Completo & 05 & 17 \\
\hline & Méd. Incompleto & 01 & 03 \\
\hline & Sup. Completo & 02 & 07 \\
\hline & Sup. Incompleto & - & - \\
\hline & Total & 30 & 100 \\
\hline \multirow{4}{*}{ Renda familiar } & Maior 1 salário mínimo & 03 & 10 \\
\hline & 1 salário mínimo & 17 & 57 \\
\hline & Menor 1 salário mínimo & 10 & 33 \\
\hline & Total & 30 & 100 \\
\hline \multirow{7}{*}{ Ocupação } & Aposentado(a) & 19 & 63 \\
\hline & Comerciante & 04 & 13 \\
\hline & Autônomo & 02 & 07 \\
\hline & Do lar & 03 & 10 \\
\hline & Professora & 01 & 3,5 \\
\hline & Ag. Comunitário de saúde & 01 & 3,5 \\
\hline & Total & 30 & 100 \\
\hline
\end{tabular}

Figura 1: Distribuição da amostra quanto ao tempo de diagnóstico da Hipertensão Arterial.

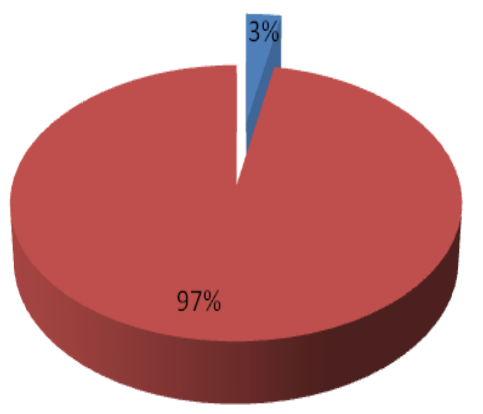

1 Ano

Mais de 1 Ano
Observa-se na Figura 1, que 29 (97\%) dos entrevistados tiveram o diagnóstico há mais de 1 ano. Entendemos que um diagnóstico cedo é de extrema importância na hipertensão arterial, pois evita assim, o aparecimento de seqüelas graves como AVC.

Diagnosticar a HAS, é importante para controlar a pressão arterial e iniciar o tratamento, seja ele medicamentoso ou não medicamentoso. Dessa maneira é de suma importância que médicos, enfermeiros e agentes comunitários de saúde acompanhem o paciente, para que este não abandone o tratamento.

Carvalho Filho, Papaléo Netto, Pasini (2005) afirmam que a hipertensão arterial é uma das maiores causas da morbimortalidade entre jovens e idosos, sendo 
responsável pelas complicações cardiovasculares, renais e oculares.

Figura 2: Distribuição da amostra em relação a situação em que foi descoberta a Hipertensão Arterial.

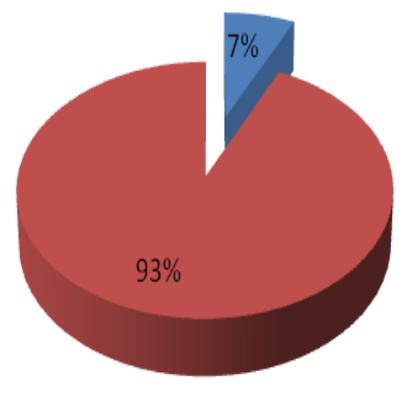

- Gestação (Pré-Eclampsia)

Através de Sintomas

De acordo com o Figura 2, observamos que 28 (97\%) dos entrevistados descobriram que eram hipertensos através da sintomatologia, que os levaram a procurar o serviço médico e nesse momento foi diagnosticada a hipertensão arterial.

Outros relataram também que descobriram ser hipertensos depois da gestação, onde tiveram préeclâmpsia e desde então, não há mais controle da pressão arterial. Os principais sintomas relatados pelos hipertensos foram: tontura,dor de cabeça, escurecimento de vista, dor no braço, vômitos e dor no peito.

Estes sintomas corroboram com a literatura já que para Smeltzer e Bare (2005) os pacientes hipertensos podem ser assintomáticos e viver por um longo tempo, ou podem ser sintomáticos e apresentar os seguintes sintomas: cefaléia, angina do peito, dor no braço, tontura, visão turva, nervosismo e vômitos.

A pré- eclampsia, segundo a VI Diretrizes de Hipertensão Arterial, é caracterizada pelo aparecimento de HAS e proteinúria ( > $300 \mathrm{mg} / 24$ h) após a $20^{\text {a }}$ semana de gestação em mulheres normotensas.

Alguns dos entrevistados também relataram ter tido algumas complicações provenientes da hipertensão, como acidente vascular cerebral e trombose.

Figura 3: Distribuição da amostra em relação a Hipertensão Arterial na família

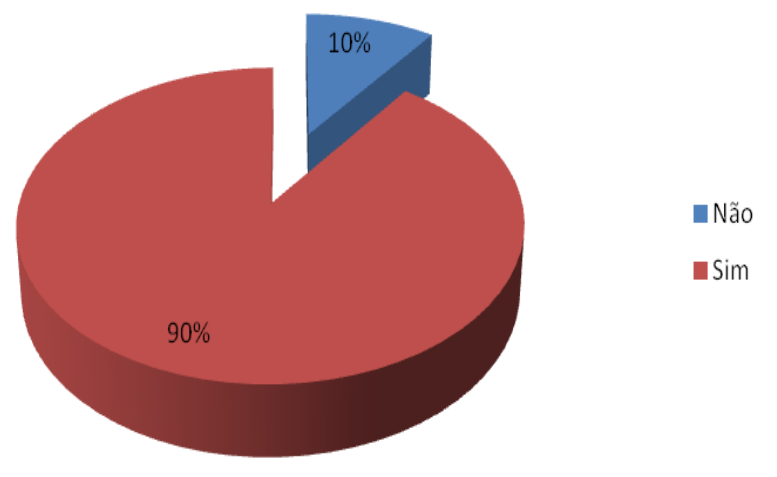

A figura 3 mostra que $27(90 \%)$ entrevistados relataram possuir familiares portadores de hipertensão arterial, onde a maioria são pais ou filhos dos mesmos.

Sabe-se que a presença de hipertensão arterial em familiares é um fator de risco considerado importante para o surgimento da doença em gerações futuras. Quanto mais próximo o grau de parentesco, maior a probabilidade (BRASILEIRO FILHO, 2000).

Para Silva e Souza (2003), a hipertensão arterial pode ser entendida como uma síndrome multifatorial, de patogênese pouco elucidada nas quais fatores genéticos e ambientais, causa a elevação constante da pressão arterial.

Cruz e Lima (1998) considera como fator de risco quem tiver pelo menos um familiar direto com problemas relacionados a HAS, como AVC, problemas cardíacos, renais ou morte súbita em idade jovem.

Para Brandão et al (2003), existe forte relação entre pressão arterial de pais e filhos, mais comum entre mães e filhos; pais com HAS determinam maior risco para seus filhos desenvolvam a HAS, necessitando de uma abordagem preventiva dessas famílias.

TABELA 2: Distribuição da amostra quanto as orientações recebidas na consulta

\begin{tabular}{lll}
\hline ORIENTAÇÕES & N & \% \\
\hline Não ingerir sal & 28 & 93 \\
Não beber & 03 & 10 \\
Não fumar & 02 & 07 \\
Praticar alguma atividade física & 12 & 40 \\
Não ingerir gordura & 20 & 67 \\
Comer frutas e verduras & 04 & 13 \\
\hline
\end{tabular}

Todos os entrevistados, relataram que quando procuraram o serviço de saúde, receberam orientações do que se deve fazer para evitar a elevação da pressão arterial. Geralmente essas informações são dadas pelo médico, enfermeiro ou até mesmo pelos agentes de saúde. Dell'acqua (1997) relata que a comunicação é um instrumento básico para a assistência de saúde, na qual pode detectar os possíveis problemas que estejam dificultando ou impedindo a compreensão da prática do auto cuidado.

Geralmente são transmitidas mais de uma orientação nas consultas. As orientações mais comuns ainda são: a não ingestão de sal, referida por 28 (93\%) dos entrevistados; a não ingestão de gordura, relatado por 20 $(60 \%)$ dos entrevistados.

Quanto ao sal, é importante mostrar que a ingestão excessiva de sal é um fator que desencadeia a HAS, tanto que se os alimentos não fossem conservados em sais de sódio e caso não se adicionasse cloreto de sódio no seu preparo, a PA possivelmente no decorrer da vida. (V DIRETRIZES BRASILEIRAS DE HIPERTENSÃO, 2006).

Ainda de acordo com a V Diretrizes Brasileiras de Hipertensão (2006), a ingestão de gordura deve ser $30 \%$ do valor total da dieta, sendo a saturada $10 \%$ e o colesterol $300 \mathrm{mg} / \mathrm{dia}$, já que o acúmulo de gordura abdominal é um fator de risco para várias doenças crônicas não transmissíveis

Segundo Araújo e Garcia (2006) todo adulto deve realizar de 30 a 45 minutos de exercício físico leve ou moderado, duas ou três vezes por semana, com pequenas mudanças no cotidiano, tais como: utilizar escadas ao invés do elevador, andar ao invés de usar o carro, praticar atividades de lazer como nadar e dançar.

Com relação ao tabagismo, Pessuto e Carvalho (1998), diz que a nicotina é prejudicial ao organismo, pois 
promove a liberação de catecolaminas, que aumentam a frequência cardíaca, a PA, a resistência vascular periférica, favorece a formação de coágulos.

As V Diretrizes Brasileiras de Hipertensão (2006) diz que o álcool em excesso, além de comprometer o sistema cardiovascular, pode causar outras doenças como cirrose hepática, pancreatite, úlceras gástricas, gastrite, neurites entre outras, porém, a causa de mortes mais comum é de origem cardiovascular.

É importante ressaltar durante a consulta o papel do sal, da gordura, da atividade física, do álcool e do tabaco no controle da HAS, claro que os profissionais devem usar uma linguagem clara e popular para melhor entendimento dos pacientes, já que a maioria possui um baixo nível de escolaridade.

Figura 4: Distribuição da amostra quanto ás adesões as orientações.

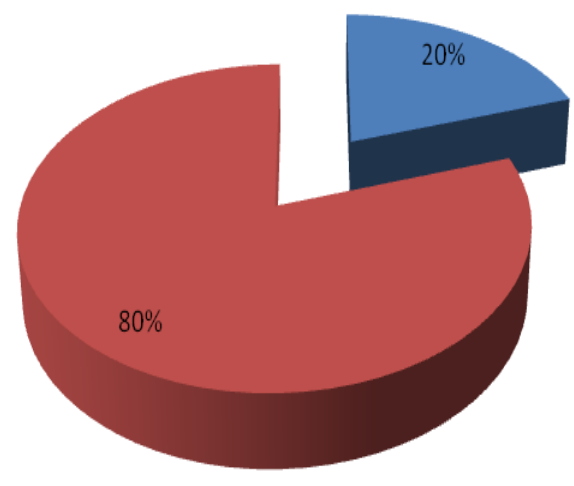

— Não

- Sim

Observando a figura 4, percebemos que uma amostra significante não segue as orientações recomendadas. A maioria dos pacientes justificou dizendo: "não gosto de comida sem sal"; "adoro comidas salgadas e gordurosas"; "gosto de tomar uma cervejinha e aí, como tudo o que não posso"; "tenho preguiça de fazer alguma atividade física". MION JR (2002) diz que uma dieta de cloreto de sódio aproximadamente $6,7 \mathrm{~g} / \mathrm{dia}$ durante 28 dias, diminui de $3,9 \mathrm{mmHg}$ na sistólica e $1,9 \mathrm{mmHg}$ na diastólica.

A dieta desempenha um papel importante no controle da hipertensão arterial. Uma dieta com teores reduzidos de cloreto de sódio, baseada em frutas, verduras e legumes, cereais integrais, leguminosas, leite e derivados desnatados, quantidades reduzidas de gorduras saturadas, trans e colesterol, mostrou ser capaz de reduzir a pressão arterial em hipertensos.

A prática de atividade física é importante no tratamento da hipertensão arterial, é o que afirma o Ministério da Saúde (BRASIL, 2006b) quando diz que os hipertensos devem realizar a prática de atividade física, pois diminui a pressão arterial e ajuda a reduzir o risco de complicações futuras.

Oliveira (2002) diz que merecem um enfoque especial as mudanças no estilo de vida, como a redução do peso, prática de atividade física, redução da ingestão do sal e álcool. Essas medidas nem sempre são bem aceitas, causando constrangimento e resistência à adesão. Daí a importância do desenvolvimento de estratégias para o controle do problema, para contribuir para uma melhor qualidade de vida e longevidade.

Araújo e Garcia (2006) afirmam que a grande maioria de pacientes hipertensos não adere à mudança do estilo de vida, já que essa mudança não é fácil, pois determina mudança de hábitos prazerosos. Acrescenta ainda que pacientes que fazem uso de medicação antihipertensiva tendem a aderir menos à mudança do estilo de vida, achando que a medicação é suficiente para o controle da doença.

Figura 5: Distribuição da amostra relacionada ao etilismo

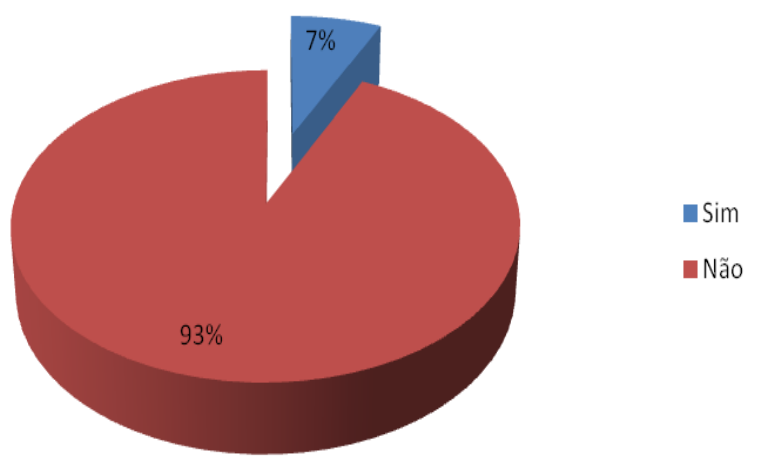

A figura 5 mostra que $7 \%$ dos entrevistados fazem uso de álcool e $93 \%$ não fazem. É um resultado satisfatório, uma vez que de acordo com Pedroso e Oliveira (2007) o paciente hipertenso deve parar de beber ou restringir a ingestão diária de álcool a uma dose de destilado de $60 \mathrm{ml}$ ou uma taça de vinho de $200 \mathrm{ml}$ ou duas latas de cerveja de $600 \mathrm{ml}$.

Conforme o Ministério da Saúde, o consumo excessivo de álcool eleva a pressão arterial e a variabilidade pressórica, aumenta a prevalência da hipertensão, é um fator de risco para acidente vascular encefálico, além de ser uma das causas de resistência terapêutica (BRASIL, 2001).

O consumo de álcool é a maior causa de adoecimento e morte no mundo, podendo ser o determinante de $10 \%$ da morbidade e mortalidade ocorridas no Brasil, tornando um dos maiores problemas de saúde pública. (BLOCH, et al, 2006).

Figura 6: Distribuição da amostra relacionada ao tabagismo.

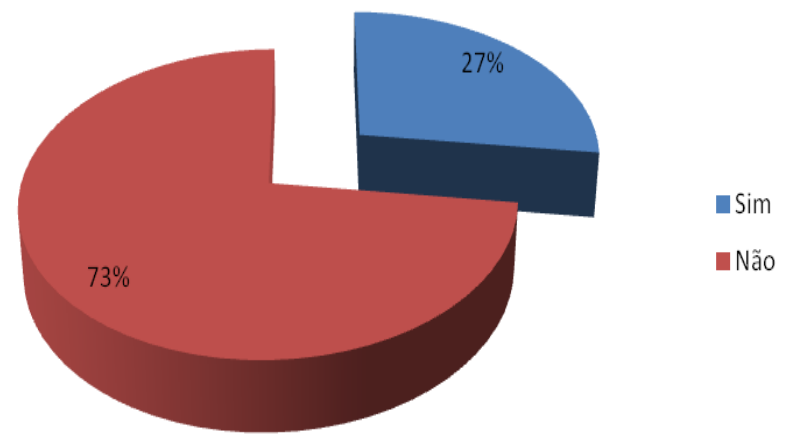

Quanto ao uso do fumo, 8 pessoas (27\%) relataram fazer uso do mesmo, como pode ser observado 
na Figura 6, em uma frequiência de 5 a 8 cigarros ao dia. Percebe-se que essas pessoas, apesar de serem hipertensas, ainda fazem uso do fumo sabendo que os fumantes correm maiores risco de apresentarem doenças respiratórias e cardiovasculares, pois o fumo dificulta o controle da pressão arterial e favorece o surgimento de complicações importantes.

De acordo com Smeltzer e Bare (2005), o tabagismo não provoca pressão arterial alta, mas se uma pessoa com hipertensão fuma, seu risco de mortalidade por cardiopatia aumenta de maneira significativa.

Em avaliação por MAPA, método que permite o registro indireto e intermitente da PA durante 24 horas ou mais enquanto o paciente realiza suas atividades habituais, a PA sistólica de hipertensos fumantes foi significadamente mais elevada do que em não- fumantes, revelando o importante efeito hipertensivo transitório do fumo (BRASIL, 2006a).

Devemos explicar que as drogas de auxílio disponíveis são essenciais no tratamento, mas que elas não substituem a tomada de uma decisão firme e persistente do próprio paciente, além do apoio de amigos e familiares.

Sobre o uso de medicação, todos os entrevistados, disseram fazer uso de medicação antihipertensiva diariamente. As drogas mais utilizadas pelos entrevistados foram: atenolol, metildolpa, clotarlidona, propranolol, enalapril, losartana potássica, captopril e hidroclorotiazida, sendo os dois últimos os mais utilizados: $20 \%$ e 17\%, respectivamente. Muitos também relataram o uso de mais de um medicamento antihipertensivo, onde a combinação mais comum foi captopril + hidroclorotiazida (10\%).

O captopril é um inibidor da enzima conversora de angiotensina que é muito eficaz em pacientes com insuficiência cardíaca, nefropatia, diabetes melitus e infarto do miocárdio (PEDROSO; OLIVEIRA, 2007).

Já para Cunningham (2001) entre as drogas mais utilizadas na hipertensão arterial, o diurético tiazídico tem demonstrado um dos melhores resultados no prognóstico cardiovascular, sendo um promotor de uma boa resposta anti-hipertensiva.

O tratamento farmacológico se impõe quando as medidas não-farmacológicas não são suficientes para o controle da pressão arterial e imediatamente, após o diagnóstico nos pacientes com alto risco cardiovascular e de hipertensão.

Para Sanjuliane (2007) o tratamento farmacológico visa, não somente o controle nas variações da P.A , como a prevenção das complicações.

\section{CONCLUSÕES}

Identificou-se que os hipertensos não seguem o tratamento, de acordo com os dados obtidos neste trabalho, embora $100 \%$ das pessoas entrevistadas façam uso de medicação anti- hipertensiva, ainda relatam uma pressão arterial elevada. Percebemos que a maioria dos entrevistados só faz uso do tratamento farmacológico regularizar a HAS, sem que haja uma interação do tratamento farmacológico e do não-farmacológico, confirmando que não basta só o tratamento medicamentoso para o controle da H.A, e sim uma mudança no estilo de vida.
Foi evidenciado, a importância das atividades educativas em saúde voltadas à essa população, transmitida de forma clara, respeitando o nível cultural de cada usuário, para que assim as informações transmitidas sejam assimiladas e praticadas. Como também é importante o desenvolvimento de ações de promoção da saúde e prevenção desta doença com os usuários ainda não acometidos ou que ainda não apresentam sintomas, uma vez que quanto mais precoce o diagnóstico, mais fácil de prevenir complicações da doença.

\section{REFERÊNCIAS BIBLIOGRÁFICAS}

ARAÚJO, C. G. S.; GARCIA, T. R. Adesão ao tratamento anti- hipertensivo: uma análise conceitual. Rev. Eletrônica de Enfermagem, Goiânia, V. 8, n. 2, 2006. Disponível em:<http://www.fen.ufg.br>.Acesso em: 08/04/2011.

BLOCK, Kátia Vergetti; RODRIGUES, Cláudia Soares; FISZEMAN, Roberto. Epidemiologia dos fatores de risco para hipertensão arterial- uma revisão crítica da literatura brasileira. Revista Brasileira de Hipertensão.v.13, n.2.São Paulo, 2006.

BRANDÃO, A. P. et al. Epidemiologia da hipertensão arterial. Rev. Soc. Cardiol. Bras. Estado de São Paulo, v. 13, n. 1, 2003 .

BRASIL. Ministério da Saúde. Instituto para o desenvolvimento da saúde. Manual de Enfermagem. Universidade de São Paulo. Brasília- DF, 2001.

BRASIL. Ministério da Saúde. Cadernos de Atenção Básica $\mathrm{n}^{\circ}$ 15. Hipertensão Arterial Sistêmica. Brasília: Ministério da Saúde, 2006a.

BRASIL. Ministério da Saúde. Secretaria de Atenção á Saúde. Cadernos de Atenção Básica: Prevenção Clínica da Doença Cardiovascular, Cerebrovascular e Renal Crônica. N¹4. Brasília: Ministério da Saúde, 2006b.

BRASILEIRO FILHO, Geraldo. Bogliolo - Patologia. 6 ed. Rio de Janeiro: Guanabara Koogan,2000

CARVALHO FILHO; PAPALÉO NETTO, Matheus; PASINI, Urbano. Hipertensão Arterial In: CARVALHO FILHO; Eurico Thomaz; PAPALÉO NETTO, Matheus. Geriatria: fundamentos, clínica e terapêutica. 2 ed. São Paulo: Atheneu, 2005.

CARVALHO, M. Hipertensão. Revista Brasileira de Hipertensão. v. 5.n.5. São Paulo: BS Cultural, 2002.

CASTRO, Maria Aurélia de et al. Necessidades humanas básicas afetadas pela hipertensão arterial e estilo de vida. RBPS, v.16, 2003. Disponível em: $<$ http//www.saude.gov.br>. Acesso em: 24/11/2010.

CRUZ, I. C. F. da; LIMA, R. Detecção dos fatores de risco para hipertensão arterial. Rev. Enferm. UERJ, Rio de Janeiro, v. 6, n. 1, 1998.

CUNNINGHAM, Susana. Hipertensão Arterial. In: GOLDMAN, Lee; BENNET, J. Claude. Cecil Tratado de 
Medicina Interna. 21 ed. Rio de Janeiro: Guanabara Koogan,2001.

DELL” AQUA, Magda Cristina Queiroz et al.Comunicação da equipe multiprofissional e indivíduos portadores de hipertensão arterial.In: Revista Latino Americana de Enfermagem.Ribeirão Preto,v.5,n.3,1997. Disponível em: <http//www.scielo.br/scielo.php script=sci_arttext pid=so0104116919970000300007\&ing=PT\&nrm=isso $>$ Acesso em:23/11/2010

LIMA, Márcia Theophilo; BUCHER, Júlia Sursis N. Ferro; LIMA, José Wellington de Oliveira. A hipertensão arterial sob o olhar de uma população carente: estudo exploratório a partir dos conhecimentos, atitudes e práticas. Cad. de Saúde Pública, v. 20 n. 4, Rio de Janeiro: jul./ago. 2004.

MARQUES, A. P. Envelhecimento da população será um desafio para a saúde pública. Publicado em16/08/2007. Disponível em:<http//www.sbh.org.br/novo/asp- publiconoticias. Acesso em: 24/11/2010

OLIVEIRA, Taciana Cavalcante de.Avaliação do processo adaptativo de um idoso portador de hipertensão arterial. In: Revista Latino- Americana de Enfermagem. Julho-Agosto,2002.

PEDROSO E; OLIVEIRA R; Blackbook- Clínica Médica. Belo Horizonte: Editora, 2007.

PESSUTO, Janete; CARVALHO, Emília Campos de. Fatores de risco em indivíduos com hipertensão arterial. In: Revista Latino-Americana de Enfermagem. v. 6; n.1. Ribeirão Preto, 1998.

SANJULIANI, A. F.A complicada relação entra a obesidade e a hipertensão arterial. Publicada em:
27/09/2007. Disponível em: <http.sbh.org/novo/asp = publico-noticias. Acesso em: 25/11/2010.

SILVA, Jorge Luis Lima; SOLANGE Lourdes de Fatores de Risco para Hipertensão Arterial Sistêmica versus estilo de vida docente.- Revista Eletrônica de Enfermagem. v. 06, n. 03, 2004. Disponível em:www.fen.ufg.br.

SMELTZER, S. C,; BARE, B. G. Brunner e Suddarth.Tratado de Enfermagem Médico-cirúrgico. $10^{a}$ ed.v.2, Rio de Janeiro: Guanabara Koogan, 2005.

SMELTZER, Susane C,; BARE,Brenda G.Brunner e Suddarth.Tratado de Enfermagem Médico-cirúrgico.10a ed.v.2,Rio de Janeiro: Guanabara Koogan, 2006.

Sociedade Brasileira de Cardiologia; Sociedade Brasileira de Hipertensão; Sociedade Brasileira de Nefrologia-SBN. V DIRETRIZES BRASILEIRAS DE HIPERTENSÃO. Arq Bras Cardiol 2006 Fev:1-48.

Sociedade Brasileira de Cardiologia; Sociedade Brasileira de Hipertensão; Sociedade Brasileira de Nefrologia. VI DIRETRIZES BRASILEIRAS DE HIPERTENSÃO ARTERIAL. Arq Bras Cardiol 2010: 95(1 supl.1): 1-51.

VICTOR, R. Hipertensão. In: GOLDMAN, L.; AUSIELLO, D. Tratado de Medicina Interna. 22a ed,.v.1.Rio de Janeiro: Elsevier, 2005.

WOODS, S. L.; FROELICHER, E. S. S.; MOTZER, S. U. Enfermagem em Cardiologia. $4^{\mathrm{a}}$ ed. Barueri: Manole, 2005.

ZAITUNE, M. P. A. et al. Hipertensão Arterial em Idosos: prevalência, fatores associados e práticas de controle no município de Campinas, São Paulo. In: Cadernos de Saúde Pública. v. 22,.n.2. São Paulo, 2006. 